\title{
Radiomic Features and Machine Learning for the Discrimination of Renal Tumor Histological Subtypes: A Pragmatic Study Using Clinical-Routine Computed Tomography
}

\author{
Johannes Uhlig ${ }^{1,2,+} \oplus$, Andreas Leha ${ }^{3,+}$, Laura M. Delonge ${ }^{1}$, Anna-Maria Haack ${ }^{1}$, Brian Shuch ${ }^{4}$, \\ Hyun S. Kim ${ }^{2,5}$, Felix Bremmer ${ }^{6}\left(\mathbb{D}\right.$, Lutz Trojan ${ }^{7}$, Joachim Lotz ${ }^{1,8}$ and Annemarie Uhlig ${ }^{4,7, *}$ \\ 1 Department of Diagnostic and Interventional Radiology, University Medical Center Goettingen, \\ Robert-Koch-Str. 40, 37075 Goettingen, Germany; johannes.uhlig@med.uni-goettingen.de (J.U.); \\ lauramaria.delonge@stud.uni-goettingen.de (L.M.D.); annamaria.haack@stud.uni-goettingen.de (A.-M.H.); \\ joachim.lotz@med.uni-goettingen.de (J.L.) \\ 2 Department of Radiology and Biomedical Imaging, Division of Interventional Radiology, Yale School of \\ Medicine, New Haven, CT 06510, USA; kevin.kim@yale.edu \\ 3 Department of Medical Statistics, University Medical Center Göttingen, Humboldtallee 32, 37073 Goettingen, \\ Germany; andreas.leha@med.uni-goettingen.de \\ 4 Institute of Urologic Oncology, David Geffen School of Medicine at UCLA, Los Angeles, CA 90095, USA; \\ bshush@mednet.ucla.edu \\ 5 Yale Cancer Center, Yale School of Medicine, New Haven, CT 06510, USA \\ 6 Institute of Pathology, University Medical Center Goettingen, Robert-Koch-Str. 40, 37075 Goettingen, \\ Germany; felix.bremmer@med.uni-goettingen.de \\ 7 Department of Urology, University Medical Center Goettingen, Robert-Koch-Str. 40, 37075 Goettingen, \\ Germany; lutz.trojan@med.uni-goettingen.de \\ 8 German Centre for Cardiovascular Research, Partnersite Goettingen, Robert-Koch-Str. 40, \\ 37075 Goettingen, Germany \\ * Correspondence: annemarie.uhlig@med.uni-goettingen.de; Tel.: +49-551-39-65891; Fax: +49-551-39-66165 \\ + These authors contributed equally to this study.
}

Received: 2 September 2020; Accepted: 14 October 2020; Published: 16 October 2020

Simple Summary: This study evaluates how advanced image analyses (radiomic features) and machine learning algorithms can help to distinguish subtypes of kidney tumors in computed tomography (CT) images, which is important for further patient treatment. For 201 patients, the image analyses showed a moderate performance, but robustly performed across various imaging centers and even in cases with suboptimal image quality. In particular, distinguishing one specific subtype of kidney tumor (oncocytomas) from other subtypes proves to be challenging. The algorithms presented in this study can help in the clinical decision-making process for kidney tumor patients, for example, to decide whether to perform kidney surgery or not.

Abstract: This study evaluates the diagnostic performance of radiomic features and machine learning algorithms for renal tumor subtype assessment in venous computed tomography (CT) studies from clinical routine. Patients undergoing surgical resection and histopathological assessment of renal tumors at a tertiary referral center between 2012 and 2019 were included. Preoperative venous-phase CTs from multiple referring imaging centers were segmented, and standardized radiomic features extracted. After preprocessing, class imbalance handling, and feature selection, machine learning algorithms were used to predict renal tumor subtypes using 10-fold cross validation, assessed as multiclass area under the curve (AUC). In total, $n=201$ patients were included (73.7\% male; mean age $66 \pm 11$ years), with $n=131$ clear cell renal cell carcinomas (ccRCC), $n=29$ papillary RCC, $n=11$ chromophobe RCC, $n=16$ oncocytomas, and $n=14$ angiomyolipomas (AML). An extreme gradient boosting algorithm demonstrated the highest accuracy (multiclass area under the curve $(A U C)=0.72$ ). 
The worst discrimination was evident for oncocytomas vs. AML and oncocytomas vs. chromophobe RCC (AUC $=0.55$ and AUC $=0.45$, respectively). In sensitivity analyses excluding oncocytomas, a random forest algorithm showed the highest accuracy, with multiclass $\mathrm{AUC}=0.78$. Radiomic feature analyses from venous-phase $\mathrm{CT}$ acquired in clinical practice with subsequent machine learning can discriminate renal tumor subtypes with moderate accuracy. The classification of oncocytomas seems to be the most complex with the lowest accuracy.

Keywords: renal cell carcinoma; computed tomography; image interpretation; computer-assisted; radiomics; machine learning

\section{Introduction}

In 2018, renal cell carcinoma (RCC) accounted for approximately 175,000 cancer-related deaths worldwide [1]. Over the last decades, RCC incidence has been increasing at an annual rate of approximately $2 \%$, which has been attributed to an earlier detection of small renal tumors through wider-spread use and technical advancements of cross-sectional imaging [2-4].

Depending on their cellular origin and behavior, renal tumors are classified into several histological subtypes with unique incidence, prognostic profile, and therapeutic options $[5,6]$. Despite the clinical implications of different renal tumor subtypes, their distinction by cross-sectional imaging remains imperfect: after surgical resection of renal tumors, approximately $20 \%$ of them are histopathologically assessed as benign, a percentage that increases up to $46.3 \%$ for renal tumors with a diameter $<1 \mathrm{~cm}[7,8]$.

One promising approach to optimize radiological assessment is the utilization of radiomic feature analyses and machine learning (ML) algorithms, which have been shown to perform well for various classification problems and imaging modalities $[9,10]$. Although some studies on advanced image analyses in the context of renal tumors have been published, most were based on dedicated renal imaging protocols with standardized acquisition schemes [11-13]. To date, there is no evidence of how digital data exploitation can be applied for renal tumor subtype assessment in a real-life scenario where renal tumors are often incidentally detected on single venous-phase computed tomography (CT) studies acquired at different imaging centers with various protocols and varying quality.

This study thus aims to assess renal tumor subtypes with radiomic feature analyses and subsequent machine learning in a pragmatic approach utilizing CT studies from clinical routine.

\section{Results}

\subsection{Study Cohort}

This study's cohort comprised $n=201$ renal tumors from the same number of patients, of which $n=73$ were female $(36.3 \%)$, and $n=121$ male $(63.7 \%)$. The median age was 66 years (inter-quartile range (IQR): 56-74 years). Histopathological analyses revealed clear cell renal cell carcinomas (ccRCC) in $n=131$ patients $(70.8 \%)$, papillary RCC in $n=29(15.7 \%)$, chromophobe RCC in $n=11(5.9 \%)$, and angiomyolipomas (AML) in $n=14(7.6 \%)$. A study flowchart is provided in Figure 1. The median of the largest three-dimensional (3D) diameter of the included renal tumors was $51.6 \mathrm{~mm}$ (IQR: 38.4-68.4 mm). The median CT slices' thickness was $2 \mathrm{~mm}$ (IQR: 1-5 mm), with a total of $n=87 \mathrm{CT}$ studies performed at a tertiary referral center $(43.4 \%)$. 


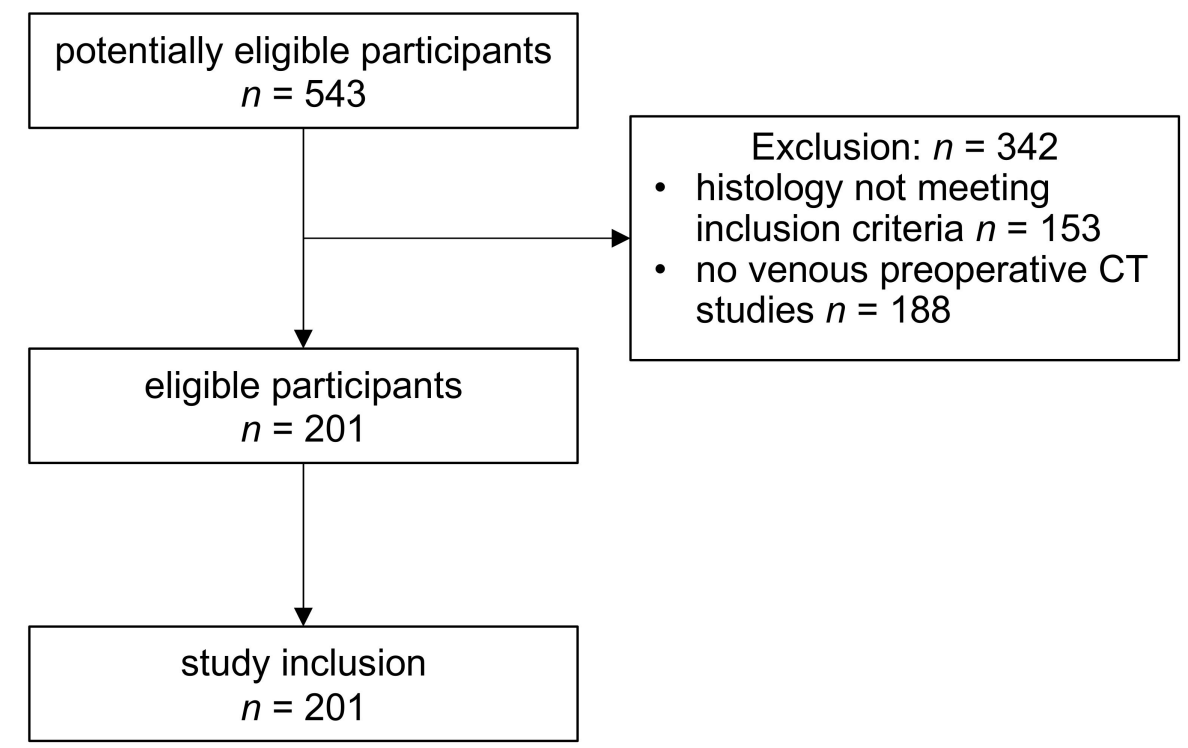

Figure 1. Flowchart of patient inclusion and exclusion.

After stratification by renal tumor histology, no statistically significant differences were evident for the maximum 3D diameter (Table 1). Patients with AML (56 \pm 14 years) or the chromophobe (60 \pm 9.2 years) subtype were younger compared to patients with the papillary (67 \pm 11 years) or ccRCC (65 \pm 11 years) subtype ( $p=0.02$ for overall difference). While ccRCC and papillary RCC were more common in male patients, female patients showed a prevalence of AMLs and chromophobe RCC (64.3\% vs. $35.7 \%$ and $63.6 \%$ vs. $36.4 \%$, respectively; $p<0.01$ for overall difference). Representative patient cases are provided in Figure 2. Additional information on histological grading and T stage of malignant cases is provided in Table S1.

Table 1. Differences in patient age, gender, and largest three-dimensional (3D) diameter according to renal tumor subtype in the full cohort without and with the synthetic minority oversampling technique (SMOTE): ccRCC, clear cell renal cell carcinomas, AML, angiomyolipomas.

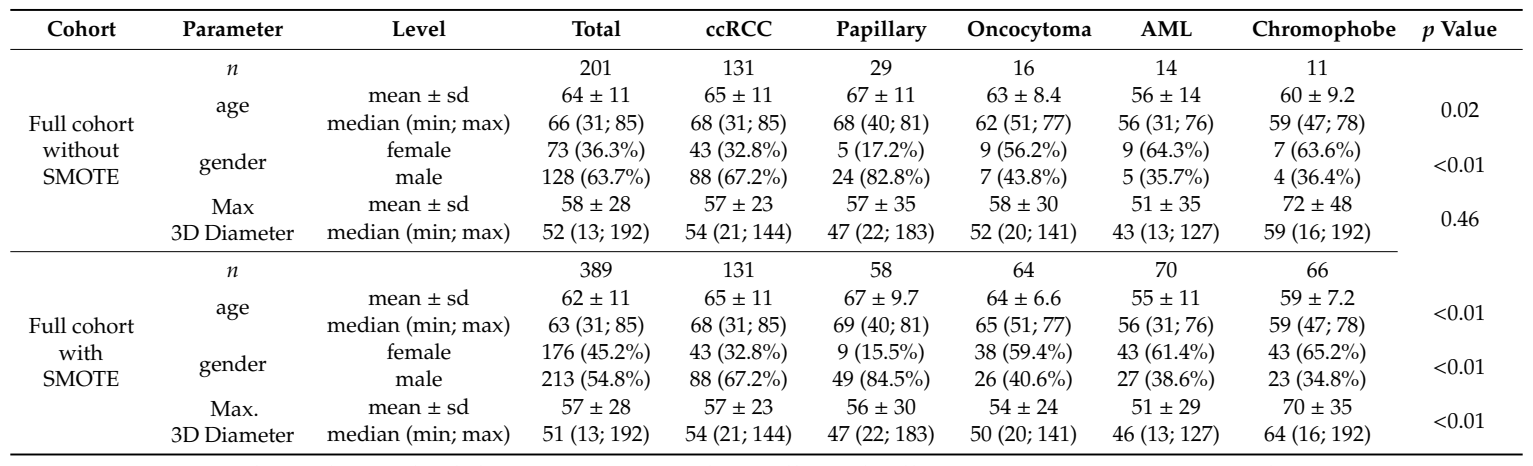

$n$ : number; sd: standard deviation; ccRCC: clear cell renal cell carcinoma; AML: angiomyolipoma. 


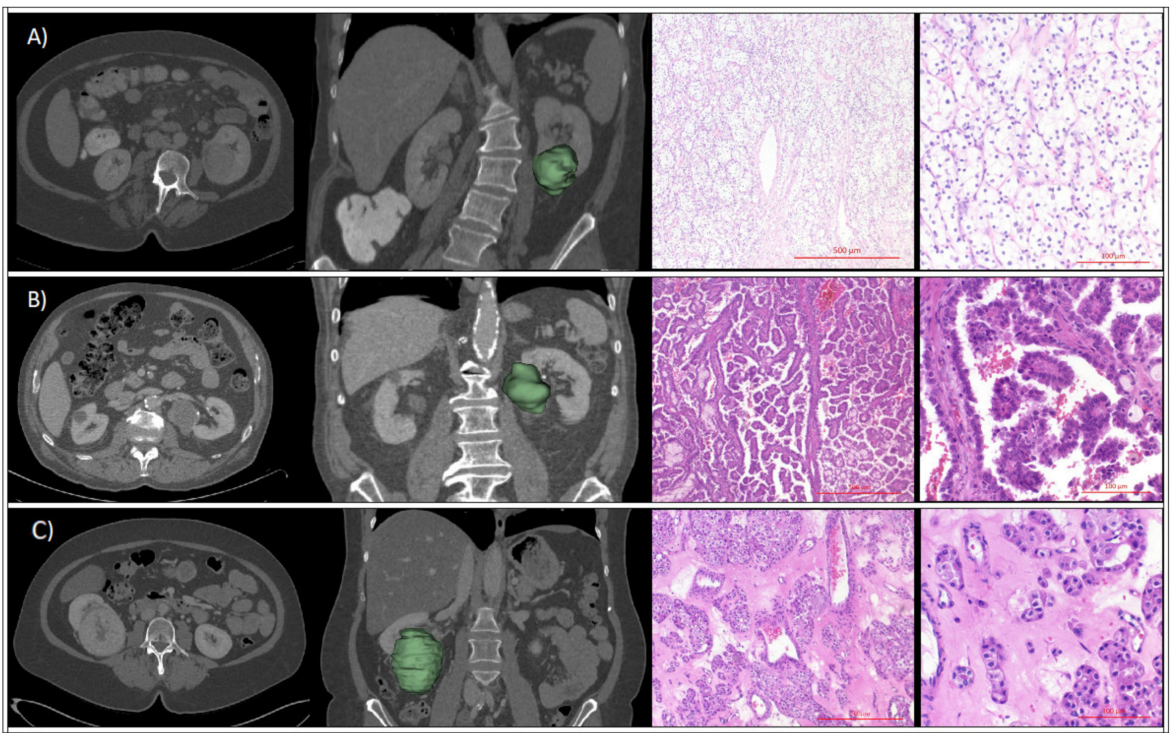

Figure 2. Clinical cases of patients presenting with: (A) left-sided renal tumor of the lower pole with heterogenous enhancement on venous-phase computed tomography $(\mathrm{CT})$ with corresponding 3D-segmentation and histopathological HE-staining at $5 \mathrm{X}$ and $20 \mathrm{X}$ magnification, which revealed a ccRCC subtype; Scale Bar $500 \mu \mathrm{m}$ and $100 \mu \mathrm{m}$; (B) left-sided central renal tumor with hypodense central attenuation, diagnosed as papillary RCC by histopathological analysis; Scale Bar $500 \mu \mathrm{m}$ and $100 \mu \mathrm{m}$; (C) right-sided renal tumor of the middle and lower pole with heterogeneous enhancement and central scar-like hypodensity, that was confirmed as oncocytoma on histopathological assessment; Scale Bar $500 \mu \mathrm{m}$ and $100 \mu \mathrm{m}$.

As summarized in Table 2 , imaging artifacts were observed in $n=60 \mathrm{CT}$ studies $(29.9 \%)$, the majority being motion artifacts $(n=56 ; 27.9 \%)$ and of moderate severity $(n=27 ; 13.4 \%)$. Metallic artifacts with severe image streaking, or combination of severe metallic and motion artifacts was evident in two cases each ( $1 \%$, respectively).

Table 2. Summary of imaging artifacts, CT slice thickness, and imaging center. IQR, inter-quartile range.

\begin{tabular}{|c|c|c|c|c|c|}
\hline Parameter & Level & Total & Any Imaging Artifacts & No Imaging Artifacts & $p$ Value \\
\hline$n$ & & 201 & 60 & 141 & \\
\hline \multirow{2}{*}{$\begin{array}{c}\text { imaging } \\
\text { center }\end{array}$} & external imaging center & $\begin{array}{c}167 \\
(83.1 \%)\end{array}$ & $58(96.7 \%)$ & $109(77.3 \%)$ & \multirow[t]{2}{*}{$<0.01$} \\
\hline & tertiary imaging center & $34(16.9 \%)$ & $2(3.3 \%)$ & $32(22.7 \%)$ & \\
\hline $\begin{array}{l}\text { slice } \\
\text { thickness }\end{array}$ & $\begin{array}{c}\text { mean } \pm \text { sd } \\
\text { median (IQR) }\end{array}$ & $2(1-5)$ & $\begin{array}{c}2.79 \pm 1.81 \\
5(4.5-5)\end{array}$ & $\begin{array}{l}4.3 \pm 1.31 \\
1.2(1-3)\end{array}$ & $<0.01$ \\
\hline
\end{tabular}

$n$ : number; sd: standard deviation; IQR: inter-quartile-range.

\subsection{Machine Learning Algorithms}

To address data imbalance, the underrepresented subtypes (chromophobe and papillary RCC, $\mathrm{AML}$, and oncocytoma) were augmented via synthetic upsampling to represent approximately $50 \%$ of the cases of the predominant renal tumor subtype ccRCC, using the synthetic minority oversampling technique (SMOTE). SMOTE generates new cases while preserving the attributes of the cohort. A summary of the patient characteristics in the SMOTE cohort is provided in Table 1.

Table 3 summarizes the diagnostic accuracy of different machine learning algorithms predicting the renal mass subtype in the full study cohort. Among the machine learning algorithms, extreme gradient boosting (xgboost) without feature selection but with upsampling achieved the numerically highest area under the curve $(\mathrm{AUC})=0.72$. Across all machine learning models, feature selection resulted in diminishing performance for all but elastic-net penalized multinomial regression (mean AUC decrease 0.04). 
Feature extraction via principle component analysis (PCA) showed mixed effects: while the average effect over all machine learning models was negative (mean AUC decrease 0.026), the ranger implementation of random forests improved. Upsampling resulted in better classification performance both with feature selection (mean AUC increase 0.06) and without feature selection (mean AUC increase 0.04).

Table 3. Area under the receiver-operating characteristics (ROC) curve (AUC) for the combinations of various machine learning models, feature selection, and imbalance handling.

\begin{tabular}{ccccccc}
\hline & \multicolumn{2}{c}{ No Upsampling } & \multicolumn{3}{c}{ SMOTE } \\
\hline Machine Learning Algorithm & No Feature Selection & RFE & PCA & No Feature Selection & RFE & PCA \\
\hline C5.0 & 0.65 & 0.58 & 0.65 & 0.71 & 0.65 & 0.63 \\
glmnet & 0.64 & 0.66 & 0.65 & 0.66 & 0.69 & 0.68 \\
knn & 0.58 & 0.57 & 0.59 & 0.59 & 0.58 & 0.56 \\
nnet & 0.63 & 0.57 & 0.60 & 0.68 & 0.67 \\
ranger & 0.68 & 0.63 & 0.70 & 0.69 & 0.64 \\
rf & 0.68 & 0.65 & 0.67 & 0.7 & 0.63 \\
svmRadial & 0.65 & 0.58 & 0.66 & 0.65 & 0.70 \\
xgboost & 0.7 & 0.62 & 0.67 & 0.72 & 0.62 & 0.67 \\
\hline
\end{tabular}

C5.0: boosted classification tree; glmnet: elastic-net penalized multinomial regression; knn: k-nearest neighbors; nnet: neural network; rf: random forest; svmRadial: support vector machine using a radial kernel; xgboost: extreme gradient boosting; ranger: random forest variant; RFE: recursive feature elimination; PCA: principle component analysis; SMOTE: synthetic minority oversampling technique.

Figure 3 shows the receiver-operating characteristics (ROC) curves for the best performing setup (xgboost in SMOTE cohort without feature selection) to differentiate the subtypes. In the underlying pairwise comparisons, the highest AUC was achieved for the differentiation of chromophobe RCCs from AMLs (AUC $=0.85$ ). The lowest AUC was evident for the differentiation of oncocytomas from AMLs and chromophobe RCCs (AUC $=0.55$ and AUC $=0.45$ ), which stipulated subgroup analyses excluding oncocytomas. All pairwise comparisons are provided in Table 4, while sensitivity and specificity of each machine learning algorithm are summarized in Table S2.

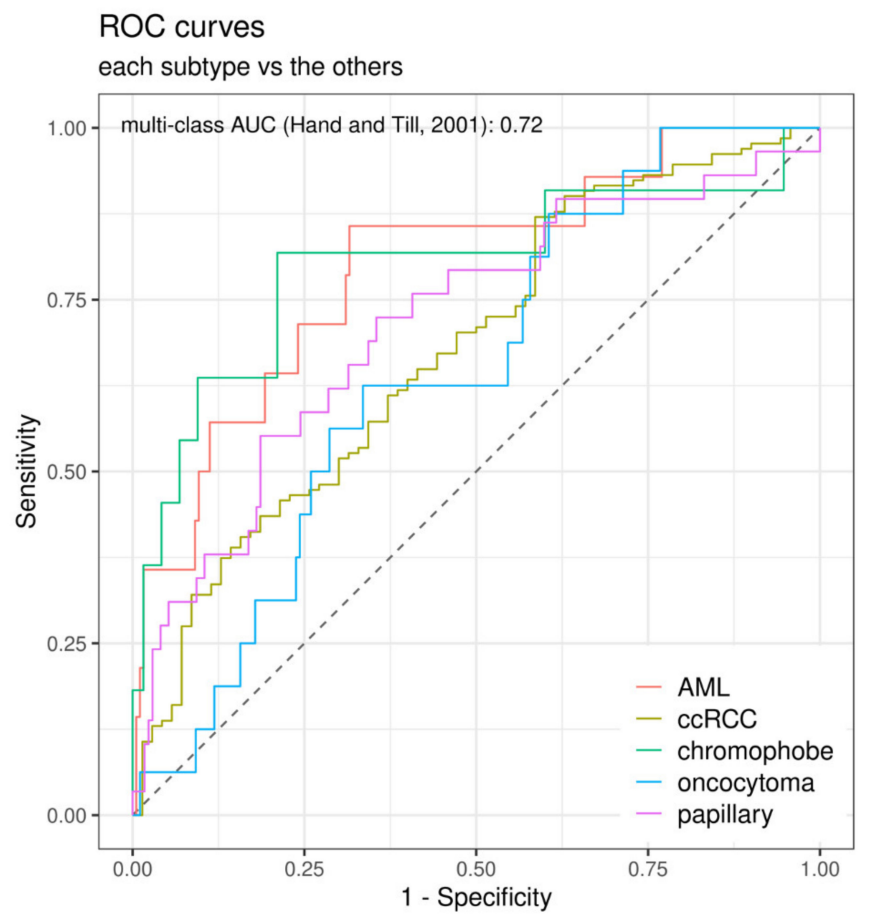

Figure 3. ROC curves for the extreme gradient boosting classifier (XG boost; with SMOTE and without feature selection). The displayed receiver-operating characteristics (ROC) curves show the discrimination of each subtype vs. the remaining ones as determined by the multi-class AUC described by Hand and Till [14]; AML: angiomyolipoma; ccRCC clear cell renal cell carcinoma; AUC: area under the ROC curve. 
Table 4. Pairwise discrimination performance of the extreme gradient boosting algorithm in the full cohort with SMOTE and without feature selection. The multi-class AUC is based on averaging AUCs from all pairwise subtype comparisons. This table shows the mean AUC from these pairwise comparisons.

\begin{tabular}{cc}
\hline Pair & AUC \\
\hline ccRCC/AML & 0.77 \\
ccRCC/chromophobe & 0.71 \\
ccRCC/oncocytoma & 0.67 \\
ccRCC/papillary & 0.67 \\
papillary/AML & 0.75 \\
papillary/chromophobe & 0.62 \\
papillary/oncocytoma & 0.74 \\
oncocytoma/AML & 0.55 \\
oncocytoma/chromophobe & 0.45 \\
AML/chromophobe & 0.85 \\
\hline
\end{tabular}

AML: angiomyolipoma; ccRCC clear cell renal cell carcinoma; AUC: area under the receiver-operating characteristics curve.

\subsection{Subgroup Analyses}

Based on the lowest AUC for the discrimination of oncocytomas vs. other subtypes in the full cohort, subgroup analyses were conducted in a subpopulation excluding patients with oncocytomas. The baseline characteristics of the subgroup are provided in in Table S3.

In the subpopulation, all machine learning models were retrained in analogy to the analyses in the full cohort. Across all machine learning models, feature selection resulted in diminishing performance, except for k-nearest neighbors $(\mathrm{knn})$ and boosted classification tree (C5.0) (mean AUC decrease 0.05). Upsampling resulted in better classification performance both with feature selection (mean AUC increase 0.05) and without feature selection (mean AUC increase 0.07). The best-performing ML algorithm in this subcohort was a random forest algorithm (ranger) using upsampling that reached a multiclass AUC $=0.78$ (Figure S1). The performance of all evaluated ML algorithms is summarized in Table S4.

In order to evaluate the influence of technical heterogeneity in the data, subgroup analyses were conducted on patients with CT scans with slice thickness $<3 \mathrm{~mm}(n=104$, Table S5), on external patients only ( $n=167$, Table S6), and on patients without imaging artifacts $(n=141$, Table S7). The highest diagnostic performance ranged from $\mathrm{AUC}=0.68$ to $\mathrm{AUC}=0.72$.

\subsection{Sensitivity Analyses}

For sensitivity analyses, cost sensitivity was introduced by setting class weights to the inverse of the class prevalence in the full study cohort. Given the multi-class approach of our clinical setting and the algorithmic architecture, weighting was not implemented for C5.0 and k-nearest neighbors $(\mathrm{knn})$. There was no relevant AUC increase compared to SMOTE, as demonstrated in Table S8.

\section{Discussion}

The accurate classification of renal tumor subtypes in imaging studies has immediate implications for clinical patient management. Still, using conventional radiological assessment strategies in CT, the discrimination of renal tumor subtypes is imperfect. In particular, incidentally detected renal tumors in CT studies that might not have been specifically tailored for renal tumor evaluation, i.e., monophasic $\mathrm{CT}$ studies, pose a radiological challenge.

In this analysis, a large cohort of renal tumor patients who underwent routine venous-phase CT studies was assessed. In a pragmatic approach, patient inclusion was not restricted regarding type of CT scanner, slice thickness, and imaging artifacts to reflect the diversity of CT imaging protocols and quality that is encountered by radiologists in everyday clinical practice. To ensure an accurate reference for further analyses, all renal tumor specimens of the included patients underwent standardized histopathological assessment. 
In this setting mimicking a real-world scenario with $\mathrm{CT}$ scans from clinical routine $\mathrm{CT}$, radiomic feature analyses and subsequent extensive machine learning algorithms were used to predict the histological subtype of renal tumors preoperatively.

Concerning demographic variables, this study's population is comparable to those described in the literature, demonstrating a male predominance of renal tumors and mean age of 64 years [5]. The lower frequency of benign renal tumors $(15 \%)$ compared to that found in the established literature (20-30\%) might well be attributable to varying characteristics of the specific subpopulation as well as to a preselection of patients for surgical resection without classic AML imaging features [7,15].

Using different approaches to imbalance handling and feature selection, the best-performing extreme gradient boosting algorithm in the full patient cohort yielded a multiclass AUC $=0.72$ for the discrimination of different renal tumor subtypes. On exploratory analyses, it was evident that the discrimination of oncocytomas from other renal tumor subtypes was the most challenging in our dataset. Indeed, in subgroup analyses excluding the oncocytoma subgroup, the ML algorithms performed better than in the full cohort, with a multiclass $\mathrm{AUC}=0.78$ for a random forest algorithm.

In contrast, the discrimination of oncocytomas from chromophobe RCCs demonstrated the lowest accuracy in our cohort (AUC $=0.45$ ). This might correlate to missing arterial-phase imaging in our study, as Amin et al. reported early contrast enhancement to be most reliable for the discrimination of oncocytomas from chromophobe RCCs [16].

Using synthetic upsampling of minority classes (SMOTE) in our study yielded superior results, which might indicate that upsampling (or weighting) of less common histological subtypes is necessary to obtain robust machine learning algorithms. In this context, it has to be highlighted that upsampling was conducted on the training datasets only, without affecting the validation datasets. Thereby, any erroneous accentuation of specific histological subtypes or imaging features by the upsampling algorithm should have negatively affected the diagnostic performance in the non-upsampled validation dataset.

The overall moderate diagnostic performance of this study might result from the heterogenous imaging data at hand, with $83.1 \%$ of the cases acquired at external imaging centers and $29.9 \%$ of imaging artifacts, which were mostly observed with higher slice thickness. These heterogenous data with sometimes low imaging quality might well have negatively affected the diagnostic performance of the machine learning algorithms.

Nevertheless, this heterogenous dataset realistically mimics the data and diagnostic challenges modern radiology faces in clinical routine. To further assess the impact of imaging quality on the diagnostic performance of machine learning algorithms, extensive subgroup analyses were conducted. These subgroups were limited to patients with high image quality, namely, those without imaging artifacts and with low slice thickness. Interestingly, the diagnostic performance in all of these scenarios was comparable to that of the full cohort, which might result from a smaller sample size. Conversely, these results indicate that the machine learning algorithms presented in this study robustly perform even in cases with low image quality.

Notably, the diagnostic performance observed in this particular study is inferior to the discrimination between benign and malignant renal tumors (AUC $=0.83$ ) that was reported by our study group for an earlier cohort of 94 patients, which outperformed the assessment by two independent radiologists (AUC $=0.68, p=0.047$ ) [17]. These differences might have been driven by the inclusion of renal tumors $>7 \mathrm{~cm}$ in diameter and a dedicated classification of oncocytomas in the current study, that proved to be most challenging.

In 2017, Coy et al. demonstrated the utility of peak lesion attenuation analyses to discriminate renal tumor histological subtypes with pairwise AUCs ranging between 0.96 and 0.79 [11]. Similar to our results, the diagnostic performance was worse for the discrimination of ccRCC from oncoytomas $(A U C=0.79)$ and of ccRCC from fat-poor AMLs (AUC $=0.83)$, indicating that these subtypes have overlapping imaging features. One explanation for the overall higher diagnostic performance of Coy et al. might be the utilization of computer-aided renal tumor segmentation and imaging during 
the corticomedullary contrast-media phase obtained from standardized CT protocols at one institution. In contrast, our patient cohort was imaged in the more readily available venous phase and manually segmented. CT studies included in our work were purposely obtained from multiple imaging centers with various CT scanners, including studies with large slice thickness and imaging artifacts. Therefore, our results might provide a more realistic estimate on how well radiomic feature analyses and ML algorithms perform in a real-world scenario in a heterogeneous patient population.

There are several published studies regarding machine learning in renal imaging [18-21]. Notably, using support vector machines (SVMs) in a cohort of $n=58$ patients, Feng et al. assessed their diagnostic potential to discriminate between RCCs and fat-poor AMLs, with an accuracy of nearly $94 \%$ [12]. Kocak et al. evaluated $n=68$ patients and described an accuracy for discrimination of non-clear-cell RCC from ccRCC of $85 \%$, while the accuracy for renal tumor subtype assessment was lower, at $69 \%$ [13]. Yu et el. evaluated renal tumors in $n=119$ patients using radiomic features and SVM and reported pairwise comparisons between selected renal tumor subtypes with AUC up to $92 \%$, although a joint model for comprehensive assessment of all renal tumor subtypes was not reported [10].

Although these studies reported promising results, they were mostly designed to only include CT studies from selected imaging centers, often with standardized multiphasic protocols. As demonstrated in our study, renal CT imaging in clinical reality varies regarding imaging quality, available slice thickness, and acquisition protocol, with several patients presenting with imaging studies from external imaging centers. Therefore, the question remains of how well the promising results from standardized imaging studies are generalizable to a broader clinical population.

The underlying biopathological changes associated with specific radiomic feature profiles of renal tumor subtypes are not fully understood yet. Some authors hypothesized that radiomic features correlate with the degree of microvessel density [22] and changes in renal cell metabolism [23-25].

This study has several limitations: first, given the geographical location of the tertiary center where renal tumor resections were performed, only patients of Caucasian race from a restricted European region were evaluated, which could limit the generalizability of the results to a more diverse population. Second, given the sample size of the evaluated patient cohort, no independent validation dataset was created, and results of the machine learning algorithms were based on cross-validation, which might have resulted in overfitted and overly optimistic results. Third, restriction of analyses to the most common renal tumor subtypes ccRCC, papillary and chromophobe RCC, AML, and oncocytoma does not reflect the clinical diversity of renal neoplasms and might thus limit the applicability of the presented results to specific cases. Further, the inclusion of patients receiving surgical resection resulted in the exclusion of those with classical imaging presentation of fat-rich AMLs who were correctly identified in CT or MRI studies. Thus, the presented algorithms might yield differing diagnostic performance in cohorts without preselection. Moreover, no substratification of type 1 and type 2 papillary RCC was conducted in this study, given the small sample size and the large number of histological subtypes already evaluated. Finally, the lack of an independent, external validation dataset might limit the generalizability of our results, although extensive measures, such as cross-validation, were applied in our study. Given this limitation, our algorithms might have been overfitted, and the generalizability of our findings to other patient populations might be poor.

The algorithm presented in this study has several potential clinical applications: these include patient stratification for immediate treatment or watchful waiting, as well as identification of patients who might benefit from additional imaging studies, such as multiphasic CT studies or magnetic resonance imaging. Further, the algorithm could aid in finding consensus in cases of discordant renal tumor assessment by two radiologists and provide a comprehensive decision support to tumor boards.

\section{Material and Methods}

This retrospective study received prior approval by the ethics committee of the University Medical Center Goettingen (No 2/4/17, approved 24 March 2017) and is compliant with the Declaration of Helsinki. Of the patients included in this study, $n=94$ were previously described in analyses that 
evaluated the diagnostic performance of radiomic features and machine learning to discriminate between malignant and benign renal tumors [17]. This study added $n=107$ patients and focused on histological subtype assessment.

\subsection{Study Cohort Selection}

Adult patients with renal tumors consecutively presenting for surgical resection between 2012 and 2019 at the University Medical Center Goettingen who had received preoperative, contrast-enhanced CT imaging in venous phase, were included in this study. Analyses were restricted to patients diagnosed with clear cell, papillary, or chromophobe RCC or AML, irrespective of tumor diameter.

Patients with diffusely infiltrative tumors (i.e., lymphoma or chronic inflammatory changes) as well as those presenting with cystic neoplasms or Bosniak IV cysts were excluded, due to concerns regarding segmentation validity.

\subsection{CT Imaging}

In a pragmatic study design, CT studies from multiple outside imaging centers and our tertiary referral center were included. CT studies were included if they were performed after administration of intravenous iodinated contrast media, acquired in venous phase, depicting the complete kidneys, and provided as axial reconstructions using a soft-tissue kernel. For the scope of this study, venous-phase imaging was defined as homogenous enhancement of the renal parenchyma. No restrictions were made regarding CT scanner type, specific acquisition protocols or reconstructions, slice thickness, or potential imaging artifacts. Imaging artifacts were categorized on a subjective Likert scale as mild, moderate, and severe.

\subsection{Radiomic Feature Analyses}

Manual renal tumor segmentation and radiomic feature analyses were performed using the open source software "3D Slicer" and the PyRadiomics plugin by a trained reader delineating the region of interest (ROI) on axial slices [26,27]. The radiomic feature subtypes evaluated in this study are detailed in Table S9, following feature definitions by the Imaging Biomarker Standardization Initiative to ensure a standardized and reproducible approach [27,28]. All radiomic feature analyses were performed using a bin width of 25. All radiomic features were one-dimensional for downstream use in machine learning models.

\subsection{Renal Tumor Assessment}

As a gold standard, all renal tumors (partial or radical nephrectomy specimens) were histopathologically assessed at the Department of Pathology, University Medical Center Goettingen, using hematoxylin-eosin staining, as well as immunostaining for cytokeratin 7, cluster of differentiation (CD) 10 and CD117, as well as vimentin. For the establishment of AML diagnosis, staining for Melan-A, human melanoma black (HMB) 45, and actin was performed.

\subsection{Machine Learning}

Radiomic features with near-zero variance were discarded from the analysis, and only one representative of variables with identical information was retained. The remaining data were screened for linear dependencies which were broken by discarding one of the columns in the linear dependent groups. The filtered dataset consisted of 127 radiomics features next to age and gender.

Internal validation using resampling was applied in order to assess the generalization performance of the applied methodology. We used a 10-fold cross-validation (CV) scheme leading to 9 folds with test sets of size 20 and 1 fold with a test set of size 21. Stratified sampling was applied to approximate the subtype distribution of renal tumors in all training and test sets. All further steps were conducted within each fold of each repetition of the CV to avoid information leakage. 
Two strategies to handle the class imbalance in the data were pursued: (1) no special handling of class imbalance and (2) SMOTE, which oversamples the minority classes using the nearest neighbors of the cases in these classes [29]. SMOTE was applied pairwise for each of the minority classes in comparison with the remaining samples. The amount of oversampling was set to achieve approximately half the number of samples in the majority class. As a sensitivity analysis (cost sensitivity), class imbalance was addressed by setting sample weights to the inverse prevalence of their class.

Three strategies for feature selection were pursued: (1) no feature selection, i.e., all features were passed to the ML models; (2) recursive feature elimination (RFE), i.e., a random forest model was fit to the data, and the features were ordered by importance in that classifier. Then, for each $n$, a classifier was trained using the top $n$ features only, and the features yielding the best performance were retained. An internal 10-fold cross-validation was used in order to avoid bias [30]; (3) feature extraction via PCA. Within each fold, a PCA was conducted on the training set, and the principle components cumulative explaining $80 \%$ of the variance were retained. The test samples were projected onto the same PCA.

Using the two strategies to handle class imbalance and the radiomics features from the two strategies to select features, the following 8 machine learning algorithms were trained (further details and tuning parameters provided in Table S10): random forest (RF), random forest ranger (ranger), extreme gradient boosting (XG boost), boosted classification trees (C5.0), elastic-net penalized multinomial regression (glmnet), support vector machine (SVM), k-nearest neighbor (Knn), and neural network (NN).

\subsection{Statistical Analyses and Diagnostic Performance Assessment}

The diagnostic performance of the ML algorithms was assessed using the AUC in a generalization to multi-class problems proposed by Hand and Till, 2001, which aggregates the AUC over all pairwise comparisons [14]. All reported diagnostic performance measures were derived from out-of-bag samples of the 10-fold cross-validation. Sensitivity and specificity in the classification of each subtype vs. the others were calculated using a fixed cutoff at 0.5 .

All statistical analyses were performed using $R$ and RStudio with the R package "caret" [31]. An alpha level of 0.05 was chosen to indicate statistical significance. All provided $p$-values are two-sided.

\section{Conclusions}

In a large, heterogenous patient cohort with venous-phase CTs from different imaging centers, radiomic feature analyses and machine learning algorithms predicted renal tumor subtypes with moderate accuracy. The discrimination of oncocytomas from other renal tumor subtypes proved to be the most challenging.

This pragmatic approach might aid in clinical patient management, stratifying patients for immediate treatment, watchful waiting, or additional imaging, including multiphasic CT studies or magnetic resonance imaging.

Supplementary Materials: The following are available online at http:/www.mdpi.com/2072-6694/12/10/3010/ s1, Figure S1: ROC curves for the random forest "ranger" classifier in the upsampled subcohort excluding oncocytomas, with SMOTE and without feature selection, Table S1: details of histological grading and T stage of malignant renal tumor subtypes, Table S2. Sensitivity/specificity in the classification of different subtypes for the combinations of various machine learning models, feature selection, and imbalance handling methods, Table S3: Differences in patient age, gender, and largest 3-dimensional (3D) diameter according to renal tumor subtype in the cohort excluding oncocytomas, without and with synthetic minority oversampling technique (SMOTE), Table S4: Area under the receiver-operating characteristics curve (AUC) for the combinations of various machine learning models, feature selection, and imbalance handling in the subgroup analysis excluding patients with oncocytomas, Table S5: Area under the receiver-operating characteristics curve (AUC) for the combinations of various machine learning models, feature selection, and imbalance handling in the subgroup analysis including only the $n=104$ patients with CT scans with slice thickness $<3 \mathrm{~mm}$, Table S6: Area under the receiver-operating characteristics curve (AUC) for the combinations of various machine learning models, feature selection, and imbalance handling in the subgroup analysis including only the $n=167$ external patients, Table S7: Area under the receiver-operating characteristics curve (AUC) for the combinations of various machine learning models, feature selection, and imbalance handling in the subgroup analysis including only 
the $n=141$ patients without artefacts in the CT scans, Table S8: AUC for the combinations of various machine learning models and feature selection with class weights set as inverse class probability, Table S9: Details of the evaluated radiomic features, Table S10: Details of the evaluated machine learning algorithms.

Author Contributions: Conceptualization, J.U., A.L., L.M.D., A.-M.H., B.S., H.S.K., F.B., L.T., J.L. and A.U.; Data curation, L.M.D., A.-M.H. and A.U.; Formal analysis, J.U., A.L., F.B. and A.U.; Funding acquisition, A.U.; Investigation, J.U., A.L., L.M.D., A.-M.H., B.S., H.S.K., F.B., L.T., J.L. and A.U.; Methodology, J.U., A.L. and A.U.; Project administration, A.U.; Resources, B.S., H.S.K., L.T. and J.L.; Supervision, B.S., H.S.K., F.B., L.T. and J.L.; Visualization, A.L. and A.U.; Writing-original draft, J.U., A.L., L.M.D., A.-M.H., B.S., H.S.K., F.B., L.T., J.L. and A.U.; Writing-review \& editing, J.U., A.L., L.M.D., A.-M.H., B.S., H.S.K., F.B., L.T., J.L. and A.U. All authors have read and agreed to the published version of the manuscript.

Funding: This research received no external funding

Conflicts of Interest: The authors declare no conflict of interest.

$\begin{array}{ll}\text { Abbreviations } \\ \text { AML } & \text { angiomyolipoma } \\ \text { AUC } & \text { area under the ROC curve } \\ \text { CD } & \text { cluster of differentiation } \\ \text { CK } & \text { cytokeratin } \\ \text { cCRCC } & \text { clear cell renal cell carcinoma } \\ \text { CT } & \text { computed tomography } \\ \text { C5.0 } & \text { boosted classification tree } \\ \text { glmnet } & \text { elastic net penalized multinomial regression } \\ \text { HE } & \text { hematoxylin-eosin } \\ \text { HMB } & \text { human melanoma black } \\ \text { ICC } & \text { interobserver correlation coefficient } \\ \text { IQR } & \text { inter-quartile range } \\ \text { KNN } & \text { k-nearest neighbor } \\ \text { nnet } & \text { neural network } \\ \text { POM } & \text { probability of malignancy } \\ \text { RCC } & \text { renal cell carcinoma } \\ \text { RF } & \text { random forest } \\ \text { RFE } & \text { recursive feature elimination } \\ \text { ROC } & \text { receiver operating characteristics } \\ \text { ROI } & \text { region of interest } \\ \text { SMOTE } & \text { synthetic minority oversampling technique } \\ \text { SVM } & \text { support vector machine } \\ \text { US } & \text { United States } \\ \text { xgboost } & \text { extreme gradient boosting }\end{array}$

\section{References}

1. Bray, F.; Me, J.F.; Soerjomataram, I.; Siegel, R.; Torre, L.A.; Jemal, A. Global cancer statistics 2018: GLOBOCAN estimates of incidence and mortality worldwide for 36 cancers in 185 countries. CA Cancer J. Clin. 2018, 68, 394-424. [CrossRef] [PubMed]

2. Hollingsworth, J.M.; Miller, D.C.; Daignault, S.; Hollenbeck, B.K. Rising Incidence of Small Renal Masses: A Need to Reassess Treatment Effect. J. Natl. Cancer Inst. 2006, 98, 1331-1334. [CrossRef] [PubMed]

3. Kane, C.J.; Mallin, K.; Ritchey, J.; Cooperberg, M.R.; Carroll, P.R. Renal cell cancer stage migration. Cancer 2008, 113, 78-83. [CrossRef]

4. Nguyen, M.M.; Gill, I.S.; Ellison, L.M. The Evolving Presentation of Renal Carcinoma in the United States: Trends From the Surveillance, Epidemiology, and End Results Program. J. Urol. 2006, 176, 2397-2400. [CrossRef]

5. $\quad$ Ljungberg, B.; Albiges, L.; Abu-Ghanem, Y.; Bensalah, K.; Dabestani, S.; Fernández-Pello, S.; Giles, R.H.; Hofmann, F.; Hora, M.; Kuczyk, M.A.; et al. European Association of Urology Guidelines on Renal Cell Carcinoma: The 2019 Update. Eur. Urol. 2019, 75, 799-810. [CrossRef] 
6. Moch, H.; Cubilla, A.L.; Humphrey, P.A.; Reuter, V.E.; Ulbright, T.M. The 2016 WHO Classification of Tumours of the Urinary System and Male Genital Organs-Part A: Renal, Penile, and Testicular Tumours. Eur. Urol. 2016, 70, 93-105. [CrossRef]

7. Frank, I.; Blute, M.L.; Cheville, J.C.; Lohse, C.M.; Weaver, A.L.; Zincke, H. Solid Renal Tumors: An Analysis of Pathological Features Related to Tumor Size. J. Urol. 2003, 170, 2217-2220. [CrossRef]

8. Johnson, D.C.; Vukina, J.; Smith, A.B.; Meyer, A.-M.; Wheeler, S.B.; Kuo, T.-M.; Tan, H.-J.; Woods, M.E.; Raynor, M.C.; Wallen, E.M.; et al. Preoperatively Misclassified, Surgically Removed Benign Renal Masses: A Systematic Review of Surgical Series and United States Population Level Burden Estimate. J. Urol. 2015, 193, 30-35. [CrossRef]

9. Juntu, J.; Sijbers, J.; De Backer, S.; Rajan, J.; Van Dyck, D. Machine learning study of several classifiers trained with texture analysis features to differentiate benign from malignant soft-tissue tumors in T1-MRI images. J. Magn. Reson. Imaging 2010, 31, 680-689. [CrossRef]

10. Yu, H.; Scalera, J.; Khalid, M.; Touret, A.-S.; Bloch, N.; Li, B.; Qureshi, M.M.; Soto, J.A.; Anderson, S.W. Texture analysis as a radiomic marker for differentiating renal tumors. Abdom. Radiol. 2017, 42, 2470-2478. [CrossRef]

11. Coy, H.; Young, J.R.; Douek, M.L.; Brown, M.S.; Sayre, J.; Raman, S.S. Quantitative computer-aided diagnostic algorithm for automated detection of peak lesion attenuation in differentiating clear cell from papillary and chromophobe renal cell carcinoma, oncocytoma, and fat-poor angiomyolipoma on multiphasic multidetector computed tomography. Abdom. Radiol. 2017, 7, 110-1928. [CrossRef]

12. Feng, Z.; Rong, P.; Cao, P.; Zhou, Q.; Zhu, W.; Yan, Z.; Liu, Q.; Wang, W. Machine learning-based quantitative texture analysis of $C T$ images of small renal masses: Differentiation of angiomyolipoma without visible fat from renal cell carcinoma. Eur. Radiol. 2017, 28, 1625-1633. [CrossRef] [PubMed]

13. Kocak, B.; Yardimci, A.H.; Bektas, C.T.; Turkcanoglu, M.H.; Erdim, C.; Yucetas, U.; Koca, S.B.; Kilickesmez, O. Textural differences between renal cell carcinoma subtypes: Machine learning-based quantitative computed tomography texture analysis with independent external validation. Eur. J. Radiol. 2018, 107, 149-157. [CrossRef] [PubMed]

14. Hand, D.J.; Till, R.J. A Simple Generalisation of the Area Under the ROC Curve for Multiple Class Classification Problems. Mach. Learn. 2001, 45, 171-186. [CrossRef]

15. Kutikov, A.; Fossett, L.K.; Ramchandani, P.; Tomaszewski, J.E.; Siegelman, E.S.; Banner, M.P.; Van Arsdalen, K.N.; Wein, A.J.; Malkowicz, S.B. Incidence of benign pathologic findings at partial nephrectomy for solitary renal mass presumed to be renal cell carcinoma on preoperative imaging. Urology 2006, 68, 737-740. [CrossRef]

16. Amin, J.; Xu, B.; Badkhshan, S.; Abbotoy, D.; Murekeyisoni, C.; Attwood, K.M.; Schwaab, T.; Hendler, C.; Petroziello, M.; Creighton, T.T.; et al. Identification and Validation of Radiographic Enhancement for Reliable Differentiation of CD117(+) Benign Renal Oncocytoma and Chromophobe Renal Cell Carcinoma. Clin. Cancer Res. 2018, 24, 3898-3907. [CrossRef]

17. Uhlig, J.; Biggemann, L.; Nietert, M.M.; Beißbarth, T.; Lotz, J.; Kim, H.S.; Trojan, L.; Uhlig, A. Discriminating malignant and benign clinical T1 renal masses on computed tomography. Medicine 2020, 99, e19725. [CrossRef]

18. Hodgdon, T.; McInnes, M.D.; Schieda, N.; Flood, T.A.; Lamb, L.; Thornhill, R. Can Quantitative CT Texture Analysis be Used to Differentiate Fat-poor Renal Angiomyolipoma from Renal Cell Carcinoma on Unenhanced CT Images? Radiology 2015, 276, 787-796. [CrossRef]

19. Lee, H.S.; Hong, H.; Jung, D.C.; Park, S.; Kim, J. Differentiation of fat-poor angiomyolipoma from clear cell renal cell carcinoma in contrast-enhanced MDCT images using quantitative feature classification. Med Phys. 2017, 44, 3604-3614. [CrossRef]

20. Erdim, C.; Yardimci, A.H.; Bektas, C.T.; Kocak, B.; Koca, S.B.; Demir, H.; Kilickesmez, O. Prediction of Benign and Malignant Solid Renal Masses: Machine Learning-Based CT Texture Analysis. Acad. Radiol. 2020, 27, 1422-1429. [CrossRef]

21. You, M.-W.; Kim, N.; Choi, H. The value of quantitative CT texture analysis in differentiation of angiomyolipoma without visible fat from clear cell renal cell carcinoma on four-phase contrast-enhanced CT images. Clin. Radiol. 2019, 74, 547-554. [CrossRef] [PubMed] 
22. Wang, J.H.; Min, P.Q.; Wang, P.J.; Cheng, W.X.; Zhang, X.H.; Wang, Y.; Zhao, X.H.; Mao, X.Q. Dynamic CT Evaluation of Tumor Vascularity in Renal Cell Carcinoma. Am. J. Roentgenol. 2006, 186, 1423-1430. [CrossRef] [PubMed]

23. Bianchi, C.; Meregalli, C.; Bombelli, S.; Di Stefano, V.; Salerno, F.; Torsello, B.; De Marco, S.; Bovo, G.; Cifola, I.; Mangano, E.; et al. The glucose and lipid metabolism reprogramming is grade-dependent in clear cell renal cell carcinoma primary cultures and is targetable to modulate cell viability and proliferation. Oncotarget 2017, 8, 113502-113515. [CrossRef] [PubMed]

24. Lucarelli, G.; Loizzo, D.; Franzin, R.; Battaglia, S.; Ferro, M.; Cantiello, F.; Castellano, G.; Bettocchi, C.; Ditonno, P.; Battaglia, M. Metabolomic insights into pathophysiological mechanisms and biomarker discovery in clear cell renal cell carcinoma. Expert Rev. Mol. Diagn. 2019, 19, 397-407. [CrossRef]

25. Lucarelli, G.; Rutigliano, M.; Sallustio, F.; Ribatti, D.; Giglio, A.; Signorile, M.L.; Grossi, V.; Sanese, P.; Napoli, A.; Maiorano, E.; et al. Integrated multi-omics characterization reveals a distinctive metabolic signature and the role of NDUFA4L2 in promoting angiogenesis, chemoresistance, and mitochondrial dysfunction in clear cell renal cell carcinoma. Aging 2018, 10, 3957-3985. [CrossRef]

26. Fedorov, A.; Beichel, R.; Kalpathy-Cramer, J.; Finet, J.; Fillion-Robin, J.-C.; Pujol, S.; Bauer, C.; Jennings, M.; Fennessy, F.; Sonka, M.; et al. 3D Slicer as an image computing platform for the Quantitative Imaging Network. Magn. Reson. Imaging 2012, 30, 1323-1341. [CrossRef]

27. Van Griethuysen, J.J.; Fedorov, A.; Parmar, C.; Hosny, A.; Aucoin, N.; Narayan, V.; Beets-Tan, R.G.; Fillion-Robin, J.-C.; Pieper, S.; Aerts, H.J.W.L. Computational Radiomics System to Decode the Radiographic Phenotype. Cancer Res. 2017, 77, e104-e107. [CrossRef]

28. Zwanenburg, A.; Leger, S.; Vallières, M.; Löck, S. Image biomarker standardisation initiative. Radiology 2020, 295, 328-338. [CrossRef]

29. Chawla, N.; Bowyer, K.W.; Hall, L.O.; Kegelmeyer, W.P. SMOTE: Synthetic Minority Over-sampling Technique. J. Artif. Intell. Res. 2002, 16, 321-357. [CrossRef]

30. Ambroise, C.; McLachlan, G.J. Selection bias in gene extraction on the basis of microarray gene-expression data. Proc. Natl. Acad. Sci. USA 2002, 99, 6562-6566. [CrossRef]

31. Kuhn, M. Building Predictive Models inRUsing thecaretPackage. J. Stat. Softw. 2008, 28, 1-26. [CrossRef]

Publisher's Note: MDPI stays neutral with regard to jurisdictional claims in published maps and institutional affiliations.

(C) 2020 by the authors. Licensee MDPI, Basel, Switzerland. This article is an open access article distributed under the terms and conditions of the Creative Commons Attribution (CC BY) license (http://creativecommons.org/licenses/by/4.0/). 